\title{
CUTANEOUS AND BONE METASTASIS OF OCCULT BREAST CANCER: CASE REPORT
}

\author{
Rafael Everton Assunção Ribeiro da Costa', Fergus Tomás Rocha de Oliveira', Eduarda Norberto Siqueira1, \\ Ana Lúcia Nascimento Araújo², Sabas Carlos Vieira³ \\ 1 Universidade Estadual do Piauí - Teresina (PI), Brazil. \\ ${ }^{2}$ Hospital São Marcos - Teresina (PI), Brazil. \\ ${ }^{3}$ Oncocenter - Teresina (PI), Brazil.
}

Introduction: Occult breast cancer $(\mathrm{OBC})$ is defined as a metastatic carcinoma that occurs mainly in the axillary lymph nodes, derived from a primary malignant breast tumor undetectable by clinical and radiological analyses. OBC is a rare disease accounting for $0.3 \%-1.0 \%$ of all breast cancers, which occurs more commonly at the age of around 55 years. The OBC represents a rare event (especially with the manifestation of systemic metastases) and a major diagnostic challenge. Thus, the aim of this study was to report a case of $\mathrm{OBC}$ with the primary manifestation of cutaneous metastases and the subsequent detection of bone metastasis. Case report: A 70-year-old female patient, G1P0A0, nonsmoker, nonalcoholic, with hypertension, and sedentary lifestyle, exhibited multiple metastatic cutaneous lesions in the left cervical region $(2 \mathrm{~cm})$, of the left breast $(3 \mathrm{~cm})$, left axilla $(0.5 \mathrm{~cm})$, left subscapular region $(3 \mathrm{~cm})$, and in the second and fifth left chirodactyls (using anastrozole for 1 month). Mammography, ultrasonography, and magnetic resonance imaging of the breast were performed, and no structural alterations were detected in any of these tests. Biopsy of the skin lesion of the left cervical region and immunohistochemistry also indicated positive estrogen receptors (ER), progesterone receptors (PR), and GATA-binding protein 3 (GATA-3; compatible with breast cancer metastasis), establishing the diagnosis of occult breast cancer with cutaneous metastasis. The use of anastrozole was maintained. The scintigraphy was performed, indicating bone metastasis in the right coastal arcs 8 and 9 considered stable in a new test performed 8 months later. All cutaneous metastatic lesions disappeared 2 years later, with the exception of a lesion in the left cervical region, where surgical resection was indicated. The study was approved by a Research Ethics Committee, under CAAE No 30154720.0.0000.5209. Conclusion: The patient exhibited an excellent response to anastrozole and is in excellent general condition with the stability of bone metastasis.

Keywords: Neoplasm Metastasis; Neoplasms; Unknown Primary; Breast Neoplasms; Medical Records. 